Algebraic $8 \mathcal{G}$ Geometric $\mathcal{T}_{\text {opology }}$

Volume 4 (2004) 31-47

Published: 24 January 2004

ATG

\title{
Large embedded balls and Heegaard genus in negative curvature
}

\author{
DAVID BACHMAN \\ DARYL COOPER \\ MATThew E. White
}

\begin{abstract}
We show if $M$ is a closed, connected, orientable, hyperbolic 3 -manifold with Heegaard genus $g$ then $g \geq \frac{1}{2} \cosh (r)$ where $r$ denotes the radius of any isometrically embedded ball in $M$. Assuming an unpublished result of Pitts and Rubinstein improves this to $g \geq \frac{1}{2} \cosh (r)+\frac{1}{2}$. We also give an upper bound on the volume in terms of the flip distance of a Heegaard splitting, and describe isoperimetric surfaces in hyperbolic balls.
\end{abstract}

AMS Classification 57M50; 57M27, 57N16

Keywords Heegaard splitting, injectivity radius

\section{Introduction}

Let $M$ denote a closed, connected, orientable 3-manifold which admits a hyperbolic structure. By Mostow rigidity the hyperbolic metric on $M$ is unique up to isometry. Thus geometric invariants of the hyperbolic metric are actually topological invariants of $M$. One may thus attempt to relate geometric invariants to other topological invariants. For example in [5] it is shown that the volume of $M$ is at most $\pi$ times the length, $L$, of any presentation of the fundamental group of $M$. A result of Lackenby, 8] shows that for alternating links the volume is bounded above and below by explicit affine functions of a certain combinatorial invariant: the twist number.

The injectivity radius of $M$ is defined to be the radius of the smallest selftangent isometrically embedded ball in $M$. In [14] White showed that that the injectivity radius of $M$ is bounded above by a function of the rank of its fundamental group (the minimum number of generators required to generate the group), see also [7] for an extension to word hyperbolic groups. Furthermore White showed in [15] that the diameter of $M$ is bounded above, and hence the injectivity radius is bounded below, in terms of $L$. 
Since the Heegaard genus is always at least as large as the rank, White's result gives a corresponding upper bound on injectivity radius in terms of Heegaard genus. A preprint by Rubinstein provides an improved estimate for the upper bound given by Heegaard genus [12. His techniques employ minimal surfaces in an intriguing way. These ideas have inspired the result presented here. In this note, we show that the radius of any isometrically embedded ball in $M$ provides a lower bound for its Heegaard genus. The precise form of our result depends on whether or not we assume an unpublished result (which we will refer to as $\mathrm{PRH}$ ) of Pitts and Rubinstein. We give two results, the sharper one of which assumes PRH. In the course of the proof we construct certain sweepouts of manifolds of negative curvature using simplicial surfaces whose area is bounded in terms of the Heegaard genus.

Theorem 1.1 Suppose that $M$ is a closed, orientable, connected Riemannian 3-manifold with all sectional curvatures less than or equal to -1 and with Heegaard genus $g$. Then

$$
g \geq \frac{\cosh (r)}{2}
$$

Assuming PRH then

$$
g \geq \frac{\cosh (r)+1}{2}
$$

Here $r$ denotes the radius of any isometrically embedded ball in $M$.

The proof also gives a new upper bound on the volume of a closed hyperbolic 3-manifold (2.5) in terms of the flip distance (see section 3) of a Heegaard splitting. We have also found it necessary to provide a statement (3.3) and proof of the fact that an equatorial disc in a hyperbolic ball has least area among surfaces which separate the ball into two sets of equal volume. This fact is certainly known to experts but does not seem to even be stated in the literature.

An interesting open question about 3-manifolds is the relationship between the Heegaard genus and the rank of the fundamental group. Clearly

$$
\text { rank } \leq \text { genus. }
$$

Boileau and Zieschang [1] give an example of a Seifert fiber space where the rank is 2 but the genus is 3 . However there is no known hyperbolic manifold for which these numbers are known to be different. Furthermore, it is difficult to compute these quantities. Thus the current result combined with 14 provides a potential method for constructing examples and suggests the following question: 
Question Given an integer $n$, is there a constant $R(n)>0$ such that for every closed hyperbolic 3-manifold with fundamental group generated by $n$ elements, there does not exist an isometrically embedded hyperbolic ball of radius greater than $R(n)$ ?

The authors would like to thank Antonio Ros for helpful comments. A proper subset of the authors wish to subtitle this paper "Big balls imply big genus."

The second author is partially supported by the NSF.

\section{Sweepouts}

\section{$2.1 \quad$ Heegaard Splittings}

A handlebody is a 3-manifold which is homeomorphic to the closure of a regular neighborhood of a graph in $\mathbb{R}^{3}$. The image of the graph under such a homeomorphism is then a spine of the handlebody. A closed surface $S$ in $M$ is a Heegaard splitting of $\mathrm{M}$ if $S$ separates $M$ into two handlebodies. We say that the genus of a 3-manifold $M$ is the minimum among the genera of all Heegaard splittings of $M$.

\subsection{Sweepouts}

Let $S$ be a connected, orientable, closed surface and $M$ a closed, orientable 3-manifold. Let $\Phi: S \times I \rightarrow M$ be a continuous map. For each $t \in I$ let $S_{t}=\Phi(S, t)$. Then $\Phi$ is a sweepout if it satisfies the following properties:

(1) $S_{0}$ and $S_{1}$ are graphs.

(2) $\Phi_{*}: H_{3}(S \times I, \partial(S \times I)) \rightarrow H_{3}\left(M, S_{0} \cup S_{1}\right)$ is an isomorphism.

We refer to condition (2) by saying that $\Phi$ is degree one. The sweepout is smooth if $\Phi$ is $C^{\infty}$. We will refer to the following statement as PRH. It has been announced by Rubinstein and Pitts [9], and a key step in the proof has been published by Colding and De Lellis in [4] where they also announce that a forthcoming paper will complete the proof.

Hypothesis 2.1 (PRH) Suppose that $M$ is a closed Riemannian 3-manifold of Heegaard genus $g$. Then for all $\epsilon>0$ there is a smooth sweepout $\Phi: S \times I \rightarrow$ $M$ by surfaces with genus $(S)=g$ and a minimal surface $V$ in $M$ of genus at most $g$ such so that area $\left(S_{t}\right) \leq \operatorname{area}(V)+\epsilon$ for all $t \in I$. We call such a sweepout an almost minimax sweepout. 
Corollary 2.2 Assume PRH. Suppose that $M$ is a closed orientable Riemannian 3-manifold of Heegaard genus $g$ such that all sectional curvatures are at most -1 . Then for every $\epsilon>0$ there is a smooth sweepout of $M$ with the property that for every $t$ we have area $\left(S_{t}\right) \leq 4 \pi(g-1)+\epsilon$.

Proof Suppose that $V$ is a minimal surface of genus at most $g$ in $M$. Since $M$ has all sectional curvatures at most -1 it follows that $V$ has intrinsic curvature at most -1 . Therefore, by the Gauss-Bonnet theorem, we have that

$$
\operatorname{area}(V) \leq 2 \pi(2 g-2) .
$$

Using almost minimax sweepouts provided by PRH gives the result.

We will now construct certain sweepouts which we may use instead of assuming $\mathrm{PRH}$. The construction is closely related to the simplicial sweepouts used by Canary and Minsky [2], 3]. In what follows, unless otherwise stated, $M$ will always denote a closed Riemannian 3-manifold with sectional curvature at most -1 .

We will use the term triangulation in this paper in a generalized sense by not requiring the boundary of a simplex to be embedded. Formally by a triangulation of a manifold $M$ we mean a CW structure on $M$ such that the corresponding CW structure on the universal cover, $\tilde{M}$, of $M$ is a simplicial complex and that the stabilizer (under the group of covering transformations) of every simplex is trivial. By the term simplex we mean the closure of a cell.

We will use the terms vertex, edge, triangle and tetrahedron for simplices of dimension 0,1,2 and 3 respectively. In particular a one-vertex triangulation of a closed orientable surface of genus $g$ consists of one 0 -cell called the vertex, $4 g-1$ one-cells whose closures are called edges, and whose interiors are disjoint and both endpoints are at the vertex, and $2 g$ two-cells whose closures are called triangles and whose interiors are disjoint and so that the frontier of every triangle consists of 3 edges.

In the constant curvature case the sweepout is by surfaces which are a union of finitely many geodesic triangles. However we allow some, or all, of the triangles in a surface to degenerate to an interval or a point. We also allow the triangles (or intervals) to be immersed instead of embedded. In a general manifold of negative sectional curvature there are no totally geodesic surfaces. Instead we use triangles that are obtained by coning a geodesic segment to a point.

A coned $n$-simplex is a continuous map $\sigma: \Delta \rightarrow M$ where $\Delta=\left(v_{0}, v_{1}, \cdots, v_{n}\right)$ is an $n$-simplex and $M$ is a smooth Riemannian manifold such that the following 
holds. If $n=0$ then every such $\sigma$ is a coned 0 -simplex. If $n=1$ we require that $\sigma:\left(v_{0}, v_{1}\right) \rightarrow M$ is a constant speed geodesic. In particular we allow the speed to be 0 in which case the image of $\sigma$ is a single point. If $n>1$ then we require:

(1) Let $\Delta^{\prime}=\left(v_{1}, \cdots, v_{n}\right)$ be the face of $\Delta$ which omits $v_{0}$, then $\sigma \mid \Delta^{\prime}$ is a coned simplex.

(2) For every $x \in \Delta^{\prime}$ if we denote by $\left(v_{0}, x\right)$ the straight line in $\Delta$ regarded as a 1 -simplex we require that $\sigma \mid\left(v_{0}, x\right)$ is a coned 1 -simplex.

We say that $\sigma$ is coned from $v_{0}$. Since distinct vertices of $\Delta$ may have the same image under $\sigma$ we should talk about a simplex being coned from a corner of its image in $M$. However, it usually causes no confusion to identify a vertex, $v_{0}$, in $\Delta$ with its image $\sigma\left(v_{0}\right)$ in $M$ and refer to the simplex as coned from $\sigma\left(v_{0}\right)$.

The map $\sigma$ is smooth. We say that the simplex is degenerate if the image of $\sigma$ has dimension less than $n$. Since $M$ has negative sectional curvature, every arc is homotopic keeping the ends fixed to a unique geodesic. Therefore, given any continuous $\phi: \Delta \rightarrow M$ one may homotop it, keeping the vertices fixed, to a unique coned simplex. Note, however, that this map depends on the ordering of the vertices of $\Delta$.

In particular a coned triangle is uniquely determined by its restriction to the boundary together with the choice of the vertex, $v_{0}$, from which the map is coned. A coned triangle is a ruled surface. Notice that we include degenerate cases: the image of a coned triangle might be a point or a geodesic segment. Furthermore even if the image has dimension two, it may be an immersed, not embedded, triangle. In the following discussion we will initially assume that all triangles are non-degenerate and then indicate the generalization to the degenerate case.

A coned simplicial surface is continuous map $\phi: S \rightarrow M$ of a closed surface, $S$, into $M$ together with a triangulation of $S$ such that for each triangle $T$ contained in $S$ there is $\psi_{T}:\left(v_{0}, v_{1}, v_{2}\right) \rightarrow T$ and a coned 2 -simplex $\sigma_{T}$ : $\left(v_{0}, v_{1}, v_{2}\right) \rightarrow M$ such that $\sigma_{T}=\phi \circ \psi_{T}$. If $T$ is an embedded 2-simplex in $S$ this is equivalent to saying that $\phi \mid T$ is a coned 2 -simplex.

The extrinsic curvature of a ruled surface is non-positive. Since all sectional curvatures of $M$ are at most -1 it follows that the curvature of the induced metric on a ruled surface in $M$ also has curvature at most -1 . The GaussBonnet theorem and the fact that the sides of a coned triangle are geodesics implies that the area of a coned triangle in $M$ is at most $\pi$. If we endow $S$ 
with the induced (pull-back) metric then area $(S) \leq n \pi$ where $n$ is the number of triangles in the triangulation of $S$.

Given a vertex $v$ of the triangulation of $S$ the angle sum $\theta(v)$ at this vertex is the sum of the angles of the corners of the triangles incident to $v$. The GaussBonnet theorem implies

$$
\int_{S} K d A+\Sigma_{v}(2 \pi-\theta(v))=2 \pi \chi(S) .
$$

The integral is over all of $S$ except the edges and vertices. On this subset the Gauss curvature of the surface $S$ is $K \leq-1$. The sum is over all vertices of this triangulation. It follows that if a triangulation has at most one vertex for which the angle sum is less than $2 \pi$ then $\operatorname{area}(S) \leq \pi(4 g-2)$.

We can extend these ideas to the case that some, or all, of the triangles are degenerate by defining the area of a degenerate triangle to be zero.

A coned simplicial sweepout is a sweepout $\Phi: S \times I \rightarrow M$ such that for every $t \in I$ the map $\Phi \mid S \times t$ is a coned simplicial surface. The following result is perhaps of independent interest.

Theorem 2.3 Suppose that $M$ is a closed orientable Riemannian 3-manifold of Heegaard genus $g$ such that all sectional curvatures are at most -1 . Then there is a coned simplicial sweepout $\Phi: S \times I \rightarrow M$ such that for every $t \in I$ the coned simplicial surface $\Phi \mid(S \times t)$ consists of at most $4 g$ triangles and has at most one vertex at which the angle sum is less than $2 \pi$. Thus the area of every surface in this sweepout is at most $\pi(4 g-2)$.

Proof Let $S$ be a Heegaard surface of genus $g$ and $H_{0}, H_{1}$ the two handlebodies of the Heegaard splitting. For $i=0,1$ choose a spine, $S_{i}$, which is a wedge of $g$ circles in the interior of $H_{i}$. We obtain a sweepout $\Psi: S \times I \rightarrow M$ in which $S_{0}$ and $S_{1}$ are these spines and for all $t \in(0,1)$ the surfaces $S_{t}$ are isotopic to $S$. Choose basepoints $p \in S$ and $q \in M$. We will homotop this sweepout to give the required coned simplicial sweepout.

If $\Psi$ is homotoped to a map $\Psi^{\prime}$ so that, at each stage of the homotopy the image of $S \times \partial I$ is a graph, then the degree does not change, so $\Psi^{\prime}$ has degree one and is thus a sweepout. Unless otherwise stated all the homotopies are of this form.

Claim 1 We can homotop $\Psi$ so that $\Psi(p \times I)=q$ and the following holds. For $i=0,1$, there is a one-vertex triangulation, $\mathcal{T}_{i}$, of $S \times i$ with vertex $p \times i$ such that $\Psi \mid S \times i$ is a coned simplicial surface and each triangle of $\mathcal{T}_{i}$ maps to 


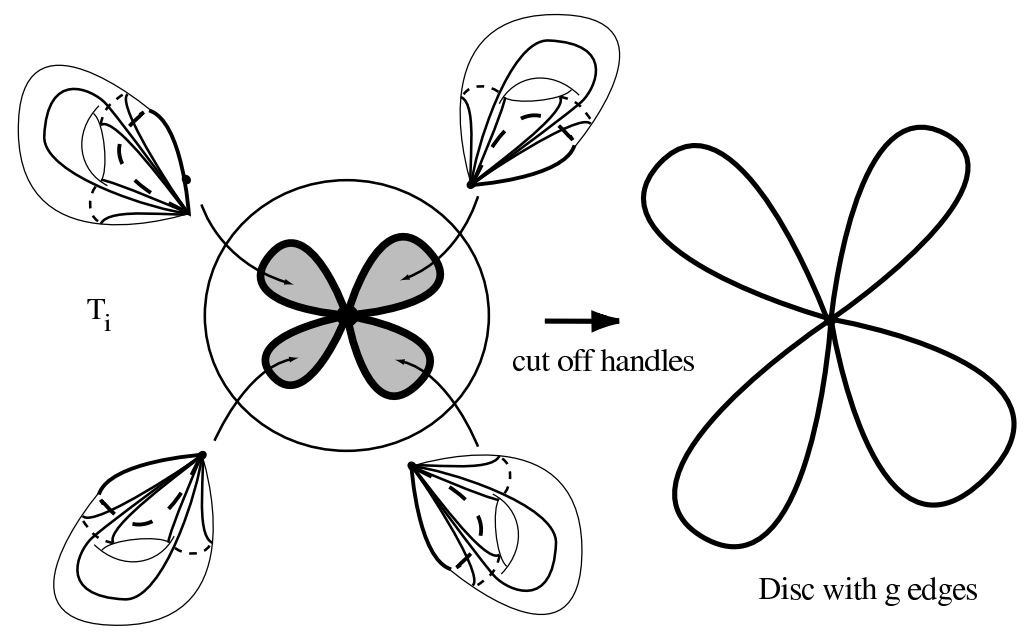

Figure 1

a (possibly immersed, possibly degenerate) geodesic arc in $M$ which starts and ends at $q$.

We may regard $\mathcal{T}_{i}$ as a triangulation of $\partial H_{i}$. The first step is to choose $\mathcal{T}_{i}$ so that there is a retraction $H_{i} \rightarrow S_{i}$ so that each triangle of $\mathcal{T}_{i}$ is mapped to either the vertex of $S_{i}$ or to an edge of $S_{i}$. The proof that this can be done follows from considering the case of genus 4 shown in Figure 1. Each handle of $H_{i}$ contributes 4 edges. The result of cutting off the handles is a disc with $g$ edges. Adding an additional $g-3$ edges decomposes this disc into $g-2$ triangles. [For genus 2 a slight variation of this is used.]

First we homotop $\Psi$ so that for $i=0,1$ the restriction $\Psi \mid S \times i$ is this retraction. Next we homotop $\Psi$ so that $p \times I$ is mapped to the basepoint $q$ and so that $S_{i}$ is homotoped to a graph $E_{i}$ in $M$ which is a wedge of geodesic arcs based at $q$. We allow the case that some of these arcs are degenerate with length zero, and that the arcs are not imbedded, only immersed, in $M$. Now we may homotop $\Psi$ as a map of pairs $(S \times I, S \times \partial I) \rightarrow\left(M, E_{1} \cup E_{2}\right)$ so that $\Psi \mid S \times i$ is a coned simplicial map with respect to the triangulation $\mathcal{T}_{i}$ for $i=0,1$. Each triangle of $\mathcal{T}_{i}$ is mapped to either the basepoint, $q$, or to an edge of $E_{i}$. We denote this new sweepout by $\Psi$. This proves claim 1 .

Claim 2 There is a map $\Psi^{\prime}: S \times I \rightarrow M$ which equals $\Psi$ on the subspace $(S \times \partial I) \cup(p \times I)$ and such that $\Psi^{\prime}$ has the property that for every $t \in I$ that $\Psi^{\prime} \mid S \times t$ is a coned simplicial surface which consists of at most $4 g$ triangles and has at most one vertex at which the angle sum is less than $2 \pi$. 
Assuming this it follows from (2.4) (and the fact that $M$ is aspherical by the Cartan-Hadamard theorem) that $\Psi^{\prime}$ is homotopic to $\Psi$ by a homotopy fixed on $S \times \partial I$. Thus $\Psi^{\prime}$ also has degree one and is therefore the required sweepout. We now prove claim 2.

It is well known, [6], that any two triangulations of a closed surface with one vertex are equivalent under isotopy and a finite sequence of flips. A flip is the following operation. Suppose $e$ is an edge of a triangulation of a surface. Then $e$ is contained in two triangles and forms the diagonal of the square formed by these triangles. Remove this diagonal and replace it with the other diagonal. This makes sense even when some boundary edges of the square are identified.
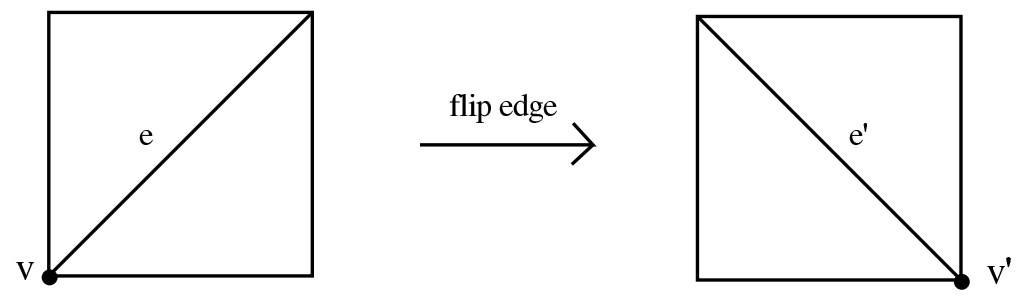

Figure 2

Let $\left\{\mathcal{T}^{i}\right\}_{i=0}^{n}$ denote a sequence of one-vertex triangulations of $S$ with the vertex at $p$ such that $\mathcal{T}^{0}=\mathcal{T}_{0}, \mathcal{T}^{n}=\mathcal{T}_{1}$, and for each $i$ the triangulations $\mathcal{T}^{i}$ and $\mathcal{T}^{i+1}$ differ by a flip. For each $0 \leq i \leq n$ homotop $\Psi \mid S \times 0$ keeping $p$ fixed to obtain a coned simplicial surface $\phi_{i}: S \rightarrow M$ with respect to the triangulation $\mathcal{T}^{i}$ such that $\phi_{i}(p)=q$. We will choose $\phi_{0}=\Psi \mid S \times 0$ and $\phi_{n}=\Psi \mid S \times 1$. Observe that $\phi_{i}$ is homotopic to $\phi_{i+1}$ keeping $p$ fixed. It is now clear that claim 2 follows from combining the homotopies given by:

Claim 3 Suppose $\mathcal{T}, T^{\prime}$ are one-vertex triangulations of $S$ each with vertex at $p$ and that $\mathcal{T}^{\prime}$ is obtained from $\mathcal{T}$ by flipping the edge $e$ of $\mathcal{T}$ to the edge $e^{\prime}$ of $\mathcal{T}^{\prime}$. Suppose that $\phi, \phi^{\prime}: S \rightarrow M$ are coned simplicial surfaces with respect to these triangulations and that they are homotopic keeping $p$ fixed, and $\phi(p)=\phi^{\prime}(p)=q$. Then there is a homotopy $H: S \times I \rightarrow M$ such that $H \mid S \times 0=\phi$ and $H \mid S \times 1=\phi^{\prime}$ and $H(p \times I)=q$. Furthermore $H$ has the property that for every $t \in I$ that $H \mid S \times t$ is a coned simplicial surface which consists of at most $4 g$ triangles and has at most one vertex at which the angle sum is less than $2 \pi$.

Let $K$ denote the union of all the edges except $e$ of $\mathcal{T}$, then $\phi\left|K=\phi^{\prime}\right| K$. Let $T$ be a triangle in $\mathcal{T}$ that does not contain $e$. Then $T$ is also a triangle in 
$\mathcal{T}^{\prime}$. If $\phi$ and $\phi^{\prime}$ both cone this triangle from the same vertex then $\phi\left|T=\phi^{\prime}\right| T$. Otherwise there is a 1-parameter family of coned simplicial surfaces connecting $\phi \mid T$ and $\phi^{\prime} \mid T$, see Figure 3. The maps in this family introduce one extra vertex, $v_{t}$, in one edge, $e$, of $T$ and two extra triangles (each of the triangles in $\mathcal{T}$ which contain $v_{t}$ in their boundary are subdivided into two triangles).

Since $M$ has non-positive curvature, given an arc $\gamma: I \rightarrow M$ there is a unique constant speed geodesic homotopic to this arc keeping endpoints fixed. Furthermore this map changes continuously as the endpoints move. One then slides the point $v_{t}$ along the edge $e=[a, c]$ starting at $a$ and ending at $c$ coning from it during this process. The angle sum at $v_{t}$ is at least $2 \pi$ because there are two edges incident to $v_{t}$ from opposite directions (their union is geodesic) cf. the proof of lemma 4.2 of [2].

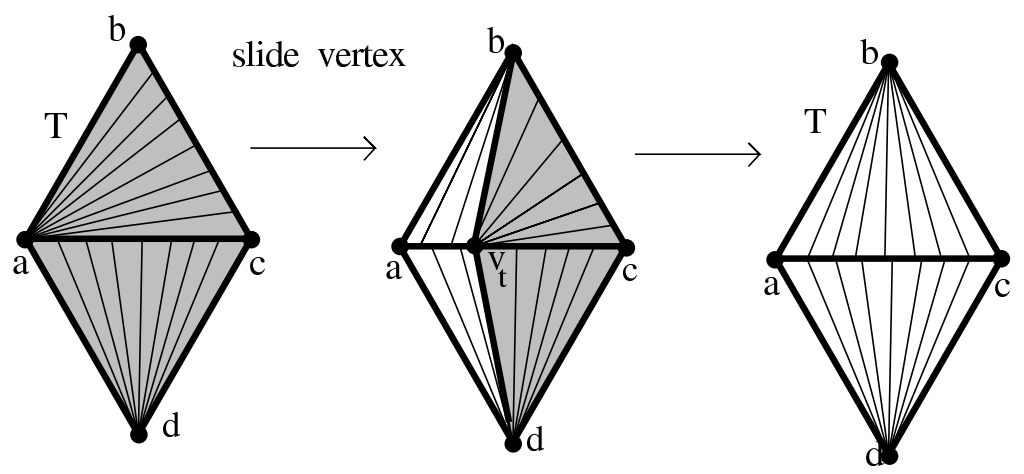

Figure 3

There are several possible variations of the picture depending on which vertex each of the two triangles is coned from. However one may view this sliding process as creating two new triangles (those to the left of $v_{t}$ shown unshaded) and enlarging them until they entirely replace the two original triangles (those to the right of $v_{t}$ shown shaded.) The vertices that the new triangles are coned from may be chosen arbitrarily. Thus one can use this process to change the vertices $T$ and $T^{\prime}$ are coned from to be any chosen vertices.

Thus by doing this process at most once for each triangle we may arrange that $\phi$ and $\phi^{\prime}$ are equal except on the interior of the quadrilateral, $Q$, which supports the flip. Let $T$ be the triangle in $\mathcal{T}$ adjacent to $Q$ along the edge $\left[v, v^{\prime}\right]$. Then $T$ is also a triangle in $\mathcal{T}^{\prime}$. We may slide vertices as above to arrange the coning in $Q \cup T$ is as shown at the left of Figure 4: thus $\phi \mid Q$ is coned from a vertex $v$ at one end of $e$. 


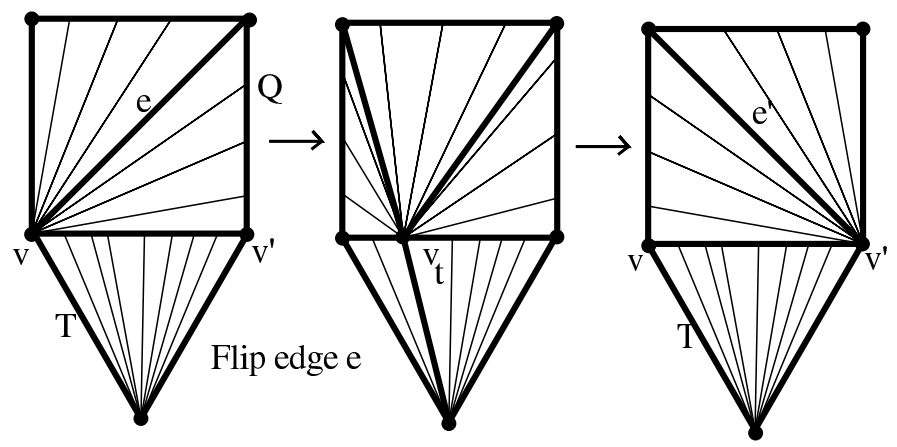

Figure 4

Now we can interpolate with a 1-parameter family of coned simplicial maps which introduce one extra vertex, $v_{t}$, and two extra triangles. As before $\theta\left(v_{t}\right) \geq$ $2 \pi$. Observe that the maps in this family do not change on $\partial Q$.

Finally we can use vertex sliding to change the way the triangles in $Q$ and $T$ are coned so that they agree with the way they are coned by $\phi^{\prime}$. This has produced a sequence of coned simplicial sweepouts interpolating between $\phi$ and $\phi^{\prime}$.

Lemma 2.4 (Homotopy lemma) Suppose that $X$ is an aspherical space and $S$ is a $C W$ complex with a single cell of dimension 0 called $p$. Suppose that $J=$ $[a, b]$ is an interval and $f_{0}, f_{1}: S \times J \rightarrow X$ are continuous and that $f_{0}\left|C=f_{1}\right| C$ where $C=(S \times \partial J) \cup(p \times J)$. Then there is a homotopy $H:(S \times J) \times I \rightarrow X$ where $I=[0,1]$ so that the following holds. For each $t \in I$ let $H_{t}: S \times J \rightarrow X$ be the map given by $H_{t}(s, j)=H(s, j, t)$ for $s \in S$ and $j \in J$ then $H_{0}=f_{0}$ and $H_{1}=f_{1}$ and $H_{t}\left|C=f_{0}\right| C$ for all $t \in I$.

Proof The hypotheses uniquely define a continuous map $H \mid D$ on

$$
D=(S \times J \times \partial I) \cup(C \times I)=[S \times \partial(J \times I)] \cup(p \times J \times I) .
$$

It suffices to show this map has a continuous extension to $S \times J \times I$. A CW structure on two spaces determines such a structure on their product. Start with the standard structure on the interval with two 0-cells and one 1-cell. Use this structure on $J$ and $I$ then the product $J \times I$ has a structure with four 0 -cells, four 1-cells and one 2-cell. We are given a cell structure on $S$ thus we get a product cell structure on $S \times J \times I$ which we now describe. For each $k$-cell $\sigma$ of $S$ there are:

(i) Four $k$-cells $\sigma \times \partial J \times \partial I$ and 
(ii) Four $(k+1)$-cells $\sigma \times(\partial J \times I)$ and $\sigma \times(J \times \partial I)$ and

(iii) One $(k+2)$-cell $\sigma \times J \times I$.

Observe that all the cells in (i) and (ii) are contained in $S \times \partial(J \times I)$ and give it a CW structure. Also $p \times J \times I$ is a cell. Thus $D$ is a subcomplex. We will extend $H$ over the cells not in $D$ in order of increasing dimension. Suppose that $\tau$ is a cell in $H$ and that $H$ has been extended over all cells of smaller dimension, hence $H$ is defined on $\partial \tau$. Since $X$ is aspherical such an extension is always possible unless dimension $(\tau)=2$. In this case we must check that $H \mid \partial \tau$ is contractible in $X$. However we claim that every cell in $S \times J \times I$ that is not contained in $D$ has dimension at least 3 . It then follows that we may extend $H$ over all cells.

Suppose that $\tau$ is a 2-cell in $S \times J \times I$ which is not contained in $D$. It follows from the above that $\tau=\sigma \times J \times I$ and $\sigma$ is a 0 -cell in $S$. This implies $\sigma=p$, but $p \times J \times I$ is contained in $D$. This proves the claim.

We remark here that when $M$ is hyperbolic, using lemmas 5.3 and 5.5 of Canary 2 one can construct a sweepout by simplicial hyperbolic surfaces with areas at most $-2 \pi \chi(S)$. However to extend this construction to the case of variable negative sectional curvature one would have to find some alternative to the proof of lemma 5.2 of that paper.

Definition Suppose that $S$ is a closed, connected, orientable surface. Let $\Gamma(S)$ be the graph whose vertices are isotopy classes of one-vertex triangulations of $S$ and two vertices in $\Gamma(S)$ are connected by an edge if there is an edge flip which converts one triangulation to the other. Given two one-vertex triangulations of $S$ the flip distance between them is the least number of edges in $\Gamma(S)$ connecting the vertices corresponding to these triangulations. We now use this to define a notion of distance for a Heegaard splitting.

Suppose $M$ is a closed 3-manifold and $S$ is a Heegaard surface for $M$ which separates $M$ into two handlebodies $H_{1}, H_{2}$. Suppose that $C_{i}$ is a spine for $H_{i}$ and that $C_{i}$ is a wedge of circles. Suppose $\mathcal{T}_{1}$ and $\mathcal{T}_{2}$ are one-vertex triangulations of $S$ so that $\mathcal{T}_{i}$ is homotopic in $H_{i}$ to $C_{i}$ such that every edge of $\mathcal{T}_{i}$ is homotoped into either the vertex of $C_{i}$ or onto one edge of $C_{i}$. Then the flip distance of the Heegaard splitting is the minimum over all such choices of the flip distance between $\mathcal{T}_{1}$ and $\mathcal{T}_{2}$.

Theorem 2.5 Suppose $M$ is a closed hyperbolic 3-manifold and $S$ is a Heegaard surface for $M$. Then the hyperbolic volume of $M$ is at most nv $v_{3}$ where $v_{3}$ is the volume of a regular ideal hyperbolic 3-simplex and $n$ is the flip distance of the Heegaard splitting. 
Sketch Proof As in the proof of (2.3), given a sequence of edge flips one constructs a map $\Phi: S \times I \rightarrow M$ which equals $\Psi$ on $S \times \partial I$. Define a sequence of spaces $N_{i}$ by $N_{0}=S \times[0,1]$ and $N_{i+1}$ is obtained from $N_{i}$ by glueing a tetrahedron along two of its faces to the quadrilateral in $\partial N_{i} \backslash(S \times 0)$ which supports the $i$ 'th edge flip. This gives a cell structure for $N_{n}$ relative to $S \times$ $I$ in which the number of tetrahedra equals the number of edge flips. Each tetrahedron is mapped by $\Psi$ as a coned (straight) map. Thus the volume of each tetrahedron is at most $v_{3}$. The map $\Psi$ is degree one by the homotopy lemma. This gives the volume bound.

It is known that there are hyperbolic 3-manifolds of Heegaard genus 2 with arbitrarily large volume; see for instance [13].

\section{Isoperimetric surfaces}

Given a metric ball, $B$, of radius $r$ in $\mathbb{H}^{3}$ an equatorial disc $\Delta$ is the intersection of a hyperbolic plane with $B$ that separates $B$ into two halves of equal volume. The area of an equatorial disc is $2 \pi(\cosh (r)-1)$.

In this section we prove that any surface which divides a hyperbolic ball into two subsets of equal volume has area at least as large as the area of an equatorial disc. The literature does not seem to contain this result exactly as stated. We present mild variations of proofs given by Antonio Ros [10, 11] to derive it from published results. We then obtain a similar result about metrics of negative sectional curvature at most -1 .

Suppose $M^{n}$ is a compact Riemannian manifold of dimension $n$ with or without boundary, and let $V(M)$ denote its $n$-dimensional Riemannian measure which we will refer to as the volume. If $\Sigma \subset M$ then the area of $\Sigma$ is the $(n-$ 1)-dimensional Riemannian measure. This area will be infinite unless $\Sigma$ has Hausdorff dimension at most $n-1$. The following existence and regularity results are cited as theorem 1 in [1] and are the culmination of work by many authors.

Theorem 3.1 Suppose $M^{n}$ is a compact Riemannian manifold of dimension $n$ with or without boundary, and let $V(M)$ denote its volume. Then for any $t$ with $0<t<V(M)$ there exists a compact region $\Omega$ whose boundary $\Sigma=\partial \Omega$ minimizes area among regions of volume $t$. Moreover, except for a closed singular set of Hausdorff dimension at most $n-8$, the boundary, $\Sigma$, of any minimizing region is a smooth embedded hypersurface with constant mean curvature, and if $\partial M \cap \Sigma \neq \phi$, then $\partial M$ and $\Sigma$ meet orthogonally. 
Definition Let $M$ be a Riemannian 3-dimensional manifold with or without boundary and volume $V(M)$. A (possibly disconnected) surface $\Sigma$ properly embedded in a 3-manifold $M$ is called isoperimetric for a volume $v<V(M)$ if

(1) $\Sigma$ encloses a region of volume $v$, and

(2) $\Sigma$ minimizes area under the constraint (1).

We shall make use of theorem 5 of [10] which we quote here for the convenience of the reader:

Theorem 3.2 (Ros) (i) If the ambient space is the sphere $M=S^{3}$ with an $O(3)$-invariant metric, then each connected component of an isoperimetric surface is a (topological) sphere of revolution.

(ii) If $M$ is a Euclidean ball with a radial metric, i.e. an $O(3)$-invariant metric, then the components of any isoperimetric surface are either spheres or discs of revolution.

A surface of revolution is a surface invariant under all isometric rotations $(\mathrm{SO}(2))$ about a fixed geodesic. We apply this to the hyperbolic metric restricted to a closed ball in hyperbolic space $\mathbb{H}^{3}$.

Theorem 3.3 Suppose that $\Omega$ is a compact subset of the closed ball, $B \equiv$ $B(r)$, of radius $r$ in $\mathbb{H}^{3}$ and let $S=\operatorname{int}(B) \cap \partial \Omega$. Suppose that $\operatorname{Volume}(\Omega)=$ $\operatorname{Volume}(B) / 2$. Then the area of $S$ is at least as large as the area of an equatorial disc. Thus

$$
\operatorname{area}(S) \geq 2 \pi(\cosh (r)-1)
$$

Proof The hyperbolic metric is homogeneous thus the existence theorem implies we may replace $S$ by an isoperimetric surface, $\Sigma$, of smaller area. Then the regularity part of the theorem implies that $\Sigma$ is a smoothly embedded surface. The hyperbolic metric restricted to $B$ is an $O(3)$-invariant metric on a Euclidean ball. Thus every component of $\Sigma$ is a disc or sphere and is a surface of revolution.

We now use an argument from the proof of theorem 2 of [10] to deduce that $\Sigma$ is a disc. First suppose that $\Sigma$ contains more than two disc components $\Delta$ and $\Delta^{\prime}$. Then applying an isometry of $B$ (element of $S O(3)$ ) we may move $\Delta$ until it first becomes tangent to $\Delta^{\prime}$ (or some other component of $\Sigma$ ) at a single point. This produces a new isoperimetric surface $\Sigma^{\prime}$ which is not regular, contradicting the theorem. Thus $\Sigma$ contains at most one disc component. A 
similar argument shows that if $\Sigma$ contains two components, one of which is a sphere, the sphere may be translated by an isometry (of $\mathbb{H}^{3}$ ) to be tangent to some other component of $\Sigma$ giving the same contradiction. Hence $\Sigma$ is connected. It remains to show $\Sigma$ is not a sphere. If it were we could translate it until it is tangent to the boundary of $B$ at a single point, again contradicting regularity. Hence $\Sigma$ is a disc of revolution.

It remains to show that this disc is flat, and therefore an equatorial disc of $B$. Since $\Sigma$ is a surface of revolution, $\partial \Sigma$ is a round circle. If this circle is the boundary of an equatorial disc, $\Delta$, then orthogonal projection onto $\Delta$ gives an area non-increasing map $\Sigma \rightarrow \Delta$, hence $\operatorname{area}(\Sigma) \geq \operatorname{area}(\Delta)$ which gives the result.

Otherwise, if $\partial \Sigma$ is not the boundary of an equatorial disk, then since it is a round circle, there is an equatorial disk $\Delta$ such that $\partial \Sigma \cap \partial \Delta=\emptyset$. We now use a (very slightly !) modified argument from the proof of theorem 5 of [1]. Let $\Omega$ be the closure of the component of $B-\Sigma$ such that $\partial \Omega$ is disjoint from $\partial \Delta$. Then we may find a metric ball $B^{\prime}$ in $\mathbb{H}^{3}$ such that:

$$
\begin{aligned}
& B^{\prime} \cap \partial B=\Omega \cap \partial B \\
& \operatorname{volume}\left(B \cap B^{\prime}\right)=\operatorname{volume}(\Omega)=\frac{1}{2} \operatorname{volume}(B) .
\end{aligned}
$$

From (2) and the fact that $\Sigma$ is isoperimetric it follows that

$$
\operatorname{area}(\Sigma) \leq \operatorname{area}\left(B \cap \partial B^{\prime}\right) .
$$

Define $W=\Omega \cup\left(B^{\prime}-B\right) \subset \mathbb{H}^{3}$ then

$$
\operatorname{area}(\partial W)=\operatorname{area}(\Sigma)+\operatorname{area}\left(\partial B^{\prime}-B\right) \leq \operatorname{area}\left(\partial B^{\prime}\right) .
$$

Now

$$
\operatorname{volume}(W)=\operatorname{volume}(\Omega)+\operatorname{volume}\left(B^{\prime}-B\right)=\operatorname{volume}\left(B^{\prime}\right) .
$$

It is an immediate consequence of (3.1) and (3.2) that given $v>0$, if a closed surface in $\mathbb{H}^{3}$ bounds a region of volume $v$ and it has least area among such surfaces then it is a hyperbolic sphere. It follows that $W$ is a metric ball in $\mathbb{H}^{3}$ and thus $W=B^{\prime}$. Hence $\Sigma=B \cap \partial B^{\prime}$ is a subset of the hyperbolic sphere $\partial B^{\prime}$.

Since $\Sigma$ is orthogonal to $\partial B$ it follows that $\partial B^{\prime}$ is orthogonal to $\partial B$. But then $\partial B^{\prime}$ must be disjoint from $\Delta$ and $B^{\prime} \cap B$ is strictly contained in one component of $B-\Delta$. This contradicts that $B^{\prime} \cap B$ has the same volume as that component. 


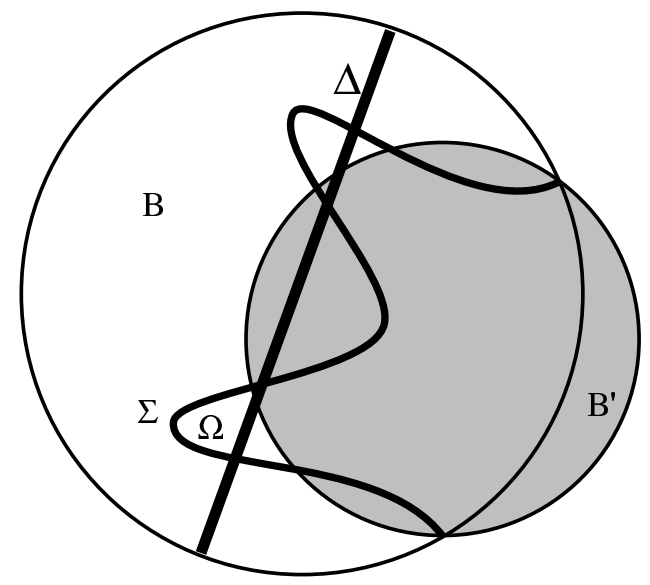

Figure 5

Corollary 3.4 Suppose that $N$ is a complete simply connected Riemannian 3-manifold with all sectional curvatures everywhere less than or equal to -1 . Let $B \equiv B_{r}(p ; N)$ be a ball of radius $r>0$ centered at some point $p \in N$. Suppose $q \in \mathbb{H}^{3}$ and let $B^{\prime}=B_{r}\left(q ; \mathbb{H}^{3}\right)$ denote the ball of radius $r$ center $q$ in $\mathbb{H}^{3}$. Let $\phi: T_{p} N \rightarrow T_{q} \mathbb{H}^{3}$ be an isometric linear map. Then the exponential maps

$$
\exp _{p}: T_{p} N \rightarrow N \quad \quad \exp _{q}: T_{q} \mathbb{H}^{3} \rightarrow \mathbb{H}^{3}
$$

are diffeomorphisms and $\phi(B)=B^{\prime}$. Suppose that $\Omega \subset B$ and that volume $(\phi \Omega)$ $=\operatorname{Volume}\left(B^{\prime}\right) / 2$. Define $S=\partial \Omega \backslash \partial B$ then area $(S) \geq 2 \pi(\cosh (r)-1)$.

Proof By the Cartan-Hadamard theorem the exponential maps are diffeomorphisms. Define $g=\exp _{q} \circ \phi \circ \exp _{p}^{-1}: N \rightarrow \mathbb{H}^{3}$. Since $\phi(0)=0$ it follows that $g(p)=q$ and since the exponential maps are isometries on one-dimensional subspaces if follows that $\phi(B)=B^{\prime}$. Since $K \leq-1$ the map $g$ is distance non-increasing. Thus it is area non-increasing, hence area $(S) \geq \operatorname{area}(g(S))$. By the theorem area $(g(S)) \geq 2 \pi(\cosh (r)-1)$.

This suggests the following:

Question Suppose that $B$ is a closed ball of radius $r$ in a complete simply connected Riemannian 3-manifold with all sectional curvatures less than or equal to -1 . Suppose that $\Sigma$ is an isoperimetric surface in $B$ which separates it into two open sets of equal volume. Is the area of $\Sigma$ at least $2 \pi(\cosh (r)-1)$ ? 
Bachman, Cooper and White

\section{Proof of Theorem (1.1)}

Theorem 4.1 Suppose that $M$ is a closed, connected, orientable Riemannian 3-manifold with all sectional curvatures at most -1. Suppose that there is a piecewise smooth sweepout of $M$ by surfaces all of which have area at most $A$. Suppose that $p \in M$ and $r=\operatorname{inj}(p)$, then

$$
2 \pi(\cosh (r)-1) \leq A .
$$

Proof We first consider the case that $M$ is hyperbolic. Let $B$ denote an isometrically embedded, standard ball of radius $r$ in $M$. We claim that there is a (possibly non-embedded) surface of area at most $A$ which separates $B$ into two open sets of equal volume. It then follows from (3.3) that $2 \pi(\cosh (r)-1) \leq$ $A$.

Let $\Phi: S \times I \rightarrow M$ be the given sweepout. Initially suppose that $\Phi \mid S \times(0,1)$ is an embedding. Consider the function $v: I \rightarrow \mathbb{R}$ defined by $v(t)=\operatorname{Volume}(B \cap$ $S[0, t])$. Then $v$ is continuous and $v(0)=0$. Since a sweepout has degree 1 it follows that $v(1)=\operatorname{volume}(B)$. Hence there is some $t=t_{0}$ such that $v\left(t_{0}\right)=$ $\frac{1}{2} \operatorname{volume}(B)$. Then $B \cap \Phi\left(S \times t_{0}\right)$ is the required surface.

The general hyperbolic case is handled as follows. The compact set $S_{t}=\Phi(S \times t)$ has finite area and therefore volume 0 . Let $U_{t}$ be a component of $M \backslash S_{t}$. Then $U_{t}$ is open and its frontier is contained in $S_{t}$. Consider $V_{t}=(S \times[0, t)) \cap \Phi^{-1}\left(U_{t}\right)$. Then $\Phi \mid: V_{t} \rightarrow U_{t}$ is proper and thus has a well defined degree. Let $\Omega(t)$ denote the closure of the union of those components $U_{t}$ for which this degree equals 1. Then $v(t)=\operatorname{Volume}(B \cap \Omega(t))$ is continuous in $t$. When $t=0$ clearly $\Omega(0)=\phi$. When $t=1$, since a sweepout has degree one $\Omega(1)=M$. Thus volume $(B \cap \Omega(1))=\operatorname{Volume}(B)$. Thus there is $t$ with $v(t)=\frac{1}{2} \operatorname{volume}(B)$. The frontier of $\Omega(t)$ is contained in $S_{t}$ and is thus a piecewise smooth surface with area at most $A$. This surface might not be embedded in $M$, however theorem (3.3) still applies (it does not assume that the boundary of the region is an embedded surface.) This completes the proof in the case that $M$ is hyperbolic.

In the variable curvature case, we first use the map $g$ from the proof of (3.4) to use the given sweepout of $M$ to construct a sweepout of a ball in $\mathbb{H}^{3}$ and then use (3.4) to obtain the result.

The proof of Theorem (1.1) is now completed by noting that the first inequality follows directly from Theorems (4.1) and (2.3), while the second follows from a combination of Theorem (4.1) and Corollary (2.2). 
Large embedded balls and Heegaard genus

\section{References}

[1] M. Boileau and H. Zieschang, Heegaard genus of closed orientable Seifert 3 manifolds, Invent. Math. vol 10, (1984), no 3, 455-468

[2] Richard D. Canary, A covering theorem for hyperbolic 3 -manifolds and its applications, Topology 35 (1996), no. 3, 751-778.

[3] Richard D. Canary and Yair Minsky, On limits of tame hyperbolic 3-manifolds, J. Differential Geom. 43 (1996), no. 1, 1-41.

[4] Tobias H. Colding and Camillo De Lellis, The min-max construction of minimal surfaces, arXiv:math.AP/0303305

[5] Daryl Cooper, The volume of a closed hyperbolic 3-manifold is bounded by $\pi$ times the length of any presentation of its fundamental group, Proc. Amer. Math. Soc. vol 127, (1999) no 3, 941-942

[6] Allen Hatcher, On triangulations of surfaces, Topology Appl. 40 (1991), no. 2, $189-194$.

[7] I. Kapovich and R. Weidmann, Nielsen Methods and Groups Acting on Hyperbolic Spaces, Geometriae Dedicata,

[8] Marc Lackenby, The volume of hyperbolic alternating link complements (2001) arXiv:math.GT/0012185, to appear in Comm. Anal. Geom.

[9] J. Pitts and J. H. Rubinstein, Applications of minimax to Minimal Surfaces and the topology of 3-manifolds, Miniconference on geometry and partial differential equations, 2 (Canberra 1986), Proc. Centre Math. Anal. Austral. Nat. Univ., 12, Austral. Nat. Univ., Canberra, (1987)

[10] Antonio Ros, The isoperimetric and Willmore problems, Global differential geometry: the mathematical legacy of Alfred Gray (Bilbao, 2000), Contemp. Math., vol 288, 149-161, Amer. Math. Soc. (2001)

[11] Antonio Ros, The Isoperimetric problem, preprint: notes of MSRI lecture series http://www.ugr.es/〜aros/isoper.htm

[12] J. H. Rubinstein, Minimal Surfaces in Geometric 3-Manifolds, preprint

[13] Jennifer Schultens, Genus 2 closed hyperbolic 3-manifolds of arbitrarily large volume, arXiv:math.GT/0206165

[14] Matthew E. White, Injectivity Radius and Fundamental Groups of Hyperbolic 3-Manifolds, Comm. in Geom. and Ann., vol 10, no 2, (2002), 377-396

[15] Matthew E. White, A diameter bound for closed, hyperbolic 3-manifolds, arXiv:math.GT/0104192

DB and MEW: Mathematics Department, Cal Poly State University

San Luis Obispo, CA 93407

DC: Mathematics Department, University of California

Santa Barbara, CA 93106, USA

Email: dbachman@calpoly.edu, cooper@math.ucsb.edu, mewhite@calpoly.edu

Received: 30 May 2003 Revised: 21 August 2003 